\title{
On a System of Equations of a Non-Newtonian Micropolar Fluid
}

\author{
G. M. de Araújo, ${ }^{1}$ M. A. F. de Araújo, ${ }^{2}$ and E. F. L. Lucena ${ }^{3}$ \\ ${ }^{1}$ Instituto de Ciências Exatas e Naturais, UFPA, Rua Augusto Corrêa s/n, 66075-110 Belém, PA, Brazil \\ ${ }^{2}$ Departamento de Matematica, UFMA, Avenida dos Portugueses 1966, 65080-805 São Luís, MA, Brazil \\ ${ }^{3}$ Departamento de Matemática, UFPA, Rua Leandro Ribeiro s/n, 68600-000 Bragança, PA, Brazil
}

Correspondence should be addressed to E. F. L. Lucena; elizardo@ufpa.br

Received 10 August 2014; Revised 8 November 2014; Accepted 16 November 2014

Academic Editor: M. Montaz Ali

Copyright (c) 2015 G. M. de Araújo et al. This is an open access article distributed under the Creative Commons Attribution License, which permits unrestricted use, distribution, and reproduction in any medium, provided the original work is properly cited.

We investigate a problem for a model of a non-Newtonian micropolar fluid coupled system. The problem has been considered in a bounded, smooth domain of $\mathbb{R}^{3}$ with Dirichlet boundary conditions. The operator stress tensor is given by $\tau(e(u))=[(\nu+$ $\left.\left.v_{0} M\left(|e(u)|^{2}\right)\right) e(u)\right]$. To prove the existence of weak solutions we use the method of Faedo-Galerkin and compactness arguments. Uniqueness and periodicity of solutions are also considered.

\section{Introduction}

Let $\Omega$ be a bounded domain in $\mathbb{R}^{d}$ with smooth boundary $\partial \Omega$, and let $T>0$. We denote by $Q_{T}$ the time space cylinder $I \times \Omega$, with lateral boundary $\Sigma=I \times \partial \Omega$, where $I=(0, T)$ is a time interval. The unsteady flows of incompressible fluids in a boundary domain $\Omega \subset \mathbb{R}^{d}, d>1$, are described by the system of equations

$$
\begin{gathered}
\rho \frac{\partial u}{\partial t}-\nabla \cdot \tau(e(u))+\rho(u \cdot \nabla) u=-\nabla p+\rho f \quad \text { in } Q_{T}, \\
\nabla \cdot u=0 \quad \text { in } Q_{T}, \\
u=0 \quad \text { on } \Sigma_{T}, \\
u(0)=u_{0} \quad \text { in } \Omega,
\end{gathered}
$$

where $u=\left(u_{1}, u_{2}, \ldots, u_{d}\right)$ is the velocity, $p$ represents the pressure, $\rho$ is a positive constant determining the density of a material, $f=\left(f_{1}, f_{2}, \ldots, f_{d}\right)$ stands for the given external body forces, $\tau: \mathbb{R}_{\text {sym }}^{d^{2}} \rightarrow \mathbb{R}_{\text {sym }}^{d^{2}}$ denotes the extra stress tensor, $e: \mathbb{R}^{d} \rightarrow \mathbb{R}_{\text {sym }}^{d^{2}}$ denotes the symmetric part of the velocity gradient; that is,

$$
e(u)=\frac{1}{2}\left[\nabla u+(\nabla u)^{T}\right]
$$

whose components are defined as in [1] by

$$
2 e_{i j}(u)=\frac{\partial u_{i}}{\partial x_{j}}+\frac{\partial u_{j}}{\partial x_{i}}, \quad i, j=1,2, \ldots, d
$$

and $\mathbb{R}_{\text {sym }}^{d^{2}}$ represents the set of all symmetric $d \times d$ matrices; that is,

$$
\mathbb{R}_{\text {sym }}^{d^{2}}=\left\{D \in \mathbb{R}^{d^{2}} ; D_{i j}=D_{j i}, i, j=1,2, \ldots, d\right\} .
$$

Note, for example, that when $\tau(e(u))$ is of the form

$$
\tau(e(u))=\mu_{0}\left(1+|e(u)|^{p-2}\right) e(u),
$$

with $p=2$, problem (1) turns into the Navier-Stokes system, which is a model for Newtonian fluids. In the expression (5), $|e(u)|$ denotes the usual Euclidean matrix norm. We observe that (5) can be written in the form

$$
\tau(e(u))=\mu_{0} M\left(|e(u)|^{2}\right) e(u),
$$

where $M: \mathbb{R}_{0}^{+} \rightarrow \mathbb{R}_{0}^{+}, M \in C^{0}(0, \infty)$ is the generalized viscosity function. Fluids constituted by (6) are sometimes named fluids with shear-dependent viscosity. Models belonging to this class of non-Newtonian fluid mechanics are frequently used in several fields of chemistry, glaciology, biology, and geology, as discussed by Malek et al. [2] . 
The first mathematical investigations of problem (1) was done by Ladyzhenskaya in 1963, where she proposed to study system (1) with (5) and $p=4$. Combining monotone operator theory and compactness arguments, she proved the existence of weak solution to model (1), if $p \geq 1+(2 d /(d+2))$, and their uniqueness if $p \geq(d+2) / 2$. See also Lions [3] for another proof of the same results. More results are known about problem (1) obtained in a series of papers, including those of Malek et al. [2], Malek et al. [4], Frehse and Málek [5], Malek et al. [1], and other mathematicians.

The equations below describe the motion of Newtonian micropolar fluids:

$$
\begin{gathered}
\frac{\partial u}{\partial t}-\left(v+v_{r}\right) \Delta u+(u \cdot \nabla) u+\nabla p=2 \nu_{r} \nabla \times w+f \quad \text { in } Q_{T} \\
\frac{\partial w}{\partial t}-\left(c_{a}+c_{d}\right) \Delta w+(u \cdot \nabla) w-\left(c_{o}+c_{d}-c_{a}\right) \nabla(\nabla \cdot w) \\
+\lambda w=2 v_{r} \nabla \times u+g \quad \text { in } Q_{T}, \\
\nabla \cdot u=0 \quad \text { in } Q_{T}, \\
u=0 \quad \text { on } \Sigma_{T}, \\
w=0 \quad \text { on } \Sigma_{T}, \\
u(0)=u_{0} \quad \text { in } \Omega, \\
w(0)=w_{0} \quad \text { in } \Omega,
\end{gathered}
$$

where $u(x, t), w(x, t) \in \mathbb{R}^{3}$ and $p(x, t) \in \mathbb{R}$, denoting for $(x, t) \in Q$, respectively, the unknown velocity, the microrotational velocity, and the hydrostatic pressure of the fluid and $\lambda$ is a positive constant. The positive constants $\nu$ and $\nu_{r}$ are, respectively, the Newtonian and microrotational viscosity. The positive constants $c_{0}, c_{a}$, and $c_{d}$ are called coefficients of angular viscosities and satisfy $c_{0}+c_{d}>c_{a}$.

The main difference with respect to modeled fluids by the Navier-Stokes is that the rotation of the particles is taken into account. The above approach was introduced by Eringen [6] The nonlinear coupled system (7) can be used to model the behavior of liquid crystals, polymeric fluids, and blood under some circumstances (see, e.g., [7]). These systems have been mainly analyzed in the book of Lukaszewicz [8].

The problem that we study in this work consists in supposing that in system (7) the fluid is of the type (5). More precisely, we investigate the mixed problem: let $\Omega$ be a bounded domain in $\mathbb{R}^{3}$ with smooth boundary $\partial \Omega$, and let $T>0$. We denote by $Q_{T}$ the time space cylinder $I \times \Omega$, with lateral boundary $\Sigma=I \times \partial \Omega$, where $I=(0, T)$ is a time interval. We find that $u, w: Q_{T} \rightarrow \mathbb{R}^{3}$ and $p: Q_{T} \rightarrow \mathbb{R}$ solving the following system of equations:

$$
\begin{gathered}
u^{\prime}-\nabla \cdot \tau(e(u))+(u \cdot \nabla) u+\nabla p=\nabla \times w+f \quad \text { in } Q_{T}, \\
w^{\prime}-v_{1} \nabla \cdot e(w)+(u \cdot \nabla) w+\lambda_{1} w=\lambda_{2} \nabla \times u+g \quad \text { in } Q_{T}, \\
\nabla \cdot u=0 \quad \text { in } Q_{T}, \\
u=0 \quad \text { on } \Sigma_{T},
\end{gathered}
$$

$$
\begin{gathered}
w=0 \quad \text { on } \Sigma_{T}, \\
u(0)=u_{0} \quad \text { in } \Omega, \\
w(0)=w_{0} \quad \text { in } \Omega,
\end{gathered}
$$

where the extra stress tensor is given by $\tau(e(u))=(\nu+$ $\left.v_{0} M\left(|e(u)|^{2}\right)\right) e(u), e(u)$ as in (2) and (3), $v_{0}, \nu_{1}, \lambda_{1}$, and $\lambda_{2}$ are positives constants, $u=\left(u_{1}, u_{2}, u_{3}\right)$, and $\nabla \times u$ is given by

$$
\nabla \times u=\left(\frac{\partial u_{3}}{\partial x_{2}}-\frac{\partial u_{2}}{\partial x_{3}}, \frac{\partial u_{1}}{\partial x_{3}}-\frac{\partial u_{3}}{\partial x_{1}}, \frac{\partial u_{2}}{\partial x_{1}}-\frac{\partial u_{1}}{\partial x_{2}}\right)
$$

the same holds for $\nabla \times w$. Let us consider $M:(0, \infty) \rightarrow$ $(0, \infty)$ satisfying the hypothesis

$$
\begin{gathered}
M \in C^{1}(0, \infty), \quad M>M_{0}>0, \quad M^{\prime}>0, \\
c_{1}|e(u)|^{2} \leq M\left(|e(u)|^{2}\right) \leq c_{2}|e(u)|^{2},
\end{gathered}
$$

where $M_{0}, c_{1}$, and $c_{2}$ are positive constants. We observe that if $M$ is a constant function, then problem (8) reduces to problem (7).

\section{Notation and Main Results}

In order to solve problem (8) we need some notations about Sobolev spaces. We use standard notation of $L^{p}(\Omega), W^{m, p}(\Omega)$, and $C^{p}(\Omega)$ for functions that are defined on $\Omega$ and range in $\mathbb{R}$ and the notation of $\mathbf{L}^{p}(\Omega), \mathbf{W}^{m, p}(\Omega)$, and $\mathbf{C}^{p}(\Omega)$ for functions that range in $\mathbb{R}^{d}$. We also work with the spaces $L^{p}\left(I ; W^{m, p}(\Omega)\right)$ or $L^{p}\left(Q_{T}\right)$.

By $\langle\cdot, \cdot\rangle$ we will represent the duality pairing between $X$ and $X^{\prime}$, with $X^{\prime}$ being the topological dual of the space $X$. We Also define the followings spaces:

$$
\mathscr{V}=\{\varphi \in \mathscr{D}(\Omega) ; \nabla \cdot \varphi=0\} .
$$

$V_{p}=V_{p}(\Omega)$ is the closure of $\mathscr{V}$ in the space $\mathbf{W}^{1, p}(\Omega), p \in$ $(1, \infty)$. In particular, $V=V_{2}$. The norm of gradient in $V_{p}$ is given by

$$
\|\nabla u\|_{p} \equiv\left[\int_{\Omega}|\nabla u(x)|^{p} d x\right]^{1 / p} .
$$

The inner product and norm in $V$ is given, respectively, by

$$
\begin{aligned}
((u, v)) & =\sum_{i, j=1}^{3} \int_{\Omega} \frac{\partial u_{i}}{\partial x_{j}}(x) \frac{\partial v_{i}}{\partial x_{j}}(x) d x, \\
\|u\|^{2} & =\sum_{i, j=1}^{3} \int_{\Omega}\left(\frac{\partial u_{i}}{\partial x_{j}}(x)\right)^{2} d x .
\end{aligned}
$$

$H=H(\Omega)$ is the closure of $\mathscr{V}$ in the space $\mathbf{L}^{2}(\Omega)$, with inner product and norm defined, respectively, by

$$
\begin{gathered}
(u, v)=\sum_{i=1}^{3} \int_{\Omega} u_{i}(x) v_{i}(x) d x, \\
|u|^{2}=\sum_{i=1}^{3} \int_{\Omega}\left|u_{i}(x)\right|^{2} d x .
\end{gathered}
$$


Remark 1. $\mathbf{H}_{0}^{1}(\Omega)$ and $\mathbf{L}^{2}(\Omega)$ are Hilbert's spaces. We note that $\mathbf{H}_{0}^{1}(\Omega) \stackrel{c}{\hookrightarrow} \mathbf{L}^{2}(\Omega) \hookrightarrow \mathbf{H}^{-1}(\Omega)$, where the first embeddings are compact.

We introduce the following bilinear and the trilinear forms, as well as the convention of summation of indices, that is, $\alpha_{i} \beta_{j}$ instead of $\sum_{i, j=1}^{d} \alpha_{i} \beta_{j}$ :

$$
\begin{aligned}
& a(u, v)=\int_{\Omega} \frac{\partial u_{i}}{\partial x_{j}}(x) \frac{\partial v_{i}}{\partial x_{j}}(x) d x=((u, v)), \quad \forall u, v \in V, \\
& b(u, v, w)=\int_{\Omega} u_{i}(x) \frac{\partial v_{j}}{\partial x_{i}}(x) w_{j}(x) d x, \quad \forall u, v, w \in V .
\end{aligned}
$$

We note that (see Lions [3])

$$
b(u, v, w)=-b(u, w, v), \quad \forall u \in V, \forall v, w \in \mathbf{H}_{0}^{1}(\Omega) .
$$

We also introduce the notations

$$
\begin{array}{cc}
A u=-\Delta u, \quad B_{u} v=(u \cdot \nabla) v, & \forall u, v \in V, \\
\mathscr{K} u=-\nabla \cdot M\left(|e(u)|^{2}\right) e(u), & \forall u \in V .
\end{array}
$$

According to this, we have

$$
\begin{gathered}
\langle A u, v\rangle=a(u, v), \quad \forall u, v \in V, \\
\left\langle B_{u} v, w\right\rangle=b(u, v, w), \quad \forall u \in V, \forall v, w \in \mathbf{H}_{0}^{1}(\Omega), \\
\langle\mathscr{K} u, v\rangle=\int_{\Omega} M\left(|e(u)|^{2}\right) e_{i j}(u) e_{i j}(v) d x, \quad \forall u, v \in V .
\end{gathered}
$$

Remark 2. We observe that $M^{\prime}>0$ implies for all $u_{1}, u_{2} \in V$ that

$$
\begin{aligned}
& \left\langle\mathscr{K} u_{1}-\mathscr{K} u_{2}, u_{1}-u_{2}\right\rangle \\
& =\int_{\Omega}\left[M\left(\left|e\left(u_{1}\right)\right|^{2}\right) e_{i j}\left(u_{1}\right)-M\left(\left|e\left(u_{2}\right)\right|^{2}\right) e_{i j}\left(u_{2}\right)\right] \\
& \quad \times\left[e_{i j}\left(u_{1}\right)-e_{i j}\left(u_{2}\right)\right] d x \geq 0 .
\end{aligned}
$$

Therefore $\mathscr{K}: V \rightarrow V^{\prime}$ is a monotonous operator.

Definition 3. Let $u_{0} \in H, w_{0} \in \mathbf{L}^{2}(\Omega), f \in L^{4 / 3}\left(I, V^{\prime}\right)$, and $g \in L^{2}\left(I ; \mathbf{H}^{-1}(\Omega)\right)$. A weak solution to (8) is a pair of functions $\{u, w\}$, such that

$$
\begin{gathered}
u \in L^{\infty}(I ; H) \cap L^{4}\left(I ; V_{4}\right) \cap L^{4}(I ; V), \\
w \in L^{\infty}\left(I ; \mathbf{L}^{2}(\Omega)\right) \cap L^{2}\left(I ; \mathbf{H}_{0}^{1}(\Omega)\right)
\end{gathered}
$$

satisfying the following identity:

$$
\begin{aligned}
\int_{0}^{T}\left\langle u^{\prime}(t), \varphi\right\rangle d t+v \int_{0}^{T} a(u(t), \varphi) d t \\
\quad+\int_{0}^{T} b(u(t), u(t), \varphi) d t \\
\quad+v_{0} \int_{0}^{T} \int_{\Omega} M\left(|e(u(t))|^{2}\right) e_{i j}(u(t)) e_{i j}(\varphi) d x d t \\
=\int_{0}^{T}(\nabla \times w(t), \varphi) d t \\
\quad+\int_{0}^{T}(f(t), \varphi) d t, \quad \forall \varphi \in \mathscr{D}(I ; \mathscr{V}), \\
\left.\quad \int_{0}^{T} \quad w^{\prime}(t), \phi\right\rangle d t+v_{1} \int_{0}^{T} a(w(t), \phi) d t \\
\quad+\int_{1}^{T}(g(t), \phi) d t, \quad \forall \phi \in \mathscr{D}(I ; \mathscr{D}(\Omega)), \\
\quad+\int_{0}^{T} b(u(t), w(t), \phi) d t+\lambda_{1} \int_{0}^{T}(w(t), \phi) d t \\
\quad \lambda_{2} \int_{0}^{T}(\nabla \times u(t), \phi) d t \\
\quad(t), \nabla \cdot \phi) d t
\end{aligned}
$$

Lemma 4 (Korn's inequality). Let $1<p<\infty$. Then, there exists a constant $K_{p}=K_{p}(\Omega)$, such that the inequality

$$
K_{p}\|v\|_{W^{1, p}(\Omega)} \leq\|e(v)\|_{L^{p}(\Omega)}
$$

is fulfilled for all $v$ satisfying either $v \in W_{0}^{1, p}(\Omega)$, where $\Omega \subset \mathbb{R}^{d}$ is open and bounded with $\partial \Omega \subset C^{1}$.

Proof. The proof of this lemma can be found in [1], page 169.

Lemma 5. Let $p \geq 2, \tau: \mathbb{R}_{\text {sym }}^{d^{2}} \rightarrow \mathbb{R}_{\text {sym }}^{d^{2}}$, and $\Phi: \mathbb{R}_{0}^{+} \rightarrow \mathbb{R}_{0}^{+}$ and the assumptions below are satisfied for all $B, D \in \mathbb{R}_{\text {sym }}^{d^{2}}$ and $i, j, k, l=1, \ldots, d$ :

$$
\begin{gathered}
\partial_{i j} \Phi\left(|D|^{2}\right)=\tau_{i j}(D), \\
\Phi(0)=\partial_{i j} \Phi(0)=0, \\
\partial_{i j} \partial_{k l} \Phi\left(|D|^{2}\right) B_{i j} B_{k l} \geq C_{1}(1+|D|)^{p-2}|B|^{2}, \\
\left|\partial_{i j} \partial_{k l} \Phi\left(|D|^{2}\right)\right| \leq C_{2}(1+|D|)^{p-2} .
\end{gathered}
$$

Then, there exist positive constants $C_{i}, i=3,4,5$, such that

$$
\begin{gathered}
C_{3}\left(1+|D|^{p-2}\right)|D|^{2} \leq \Phi\left(|D|^{2}\right) \leq C_{4}(1+|D|)^{p}, \\
(\tau(B)-\tau(D)) \cdot(B-D) \geq C_{5}|B-D|^{2} .
\end{gathered}
$$


Proof. The proof of this lemma can be found in [4], page 263.

Lemma 6 (Vitali). Let $\Omega$ be a bounded domain in $\mathbb{R}^{n}$ and $f^{m}$ : $\Omega \rightarrow \mathbb{R}$ integrable for every $m \in \mathbb{N}$. Assume that

(1) $\lim _{m \rightarrow \infty} f^{m}(x)$ exists and is finite for almost all $x \in \Omega$;

(2) for every $\varepsilon>0$ there exists $\delta>0$ such that

$$
\sup _{m \in \mathbb{N}} \int_{H}\left|f^{m}(x)\right| d x<\varepsilon, \quad \forall H \in \Omega,|H|<\delta ;
$$

then

$$
\lim _{m \rightarrow \infty} \int_{\Omega} f^{m}(x) d x=\int_{\Omega} \lim _{m \rightarrow \infty} f^{m}(x) d x .
$$

Proof. The proof of this lemma can be found in [9], page 63.

Lemma 7. Consider $d \geq 3$ and $s, r \in \mathbb{R}$, with $s>2, r>d$, verifying $(2 / s)+(d / r)=1$. If $u \in \mathbf{L}^{r}(\Omega)$, then

$$
|b(u, v, w)| \leq c\|u\|_{L^{r}(\Omega)}\|v\||w|^{2 / s}\|w\|^{d / r}
$$

for all $v, w \in V$, where $c \geq 0$ is a constant independent of $u, v$, and $w$.

Proof. The proof of this lemma can be found in [3], page 84 .

Theorem 8. If $d \leq 3, u_{0} \in H, w_{0} \in \mathbf{L}^{2}(\Omega), f \in L^{4 / 3}\left(I ; V^{\prime}\right)$, and $g \in L^{2}\left(I ; \mathbf{H}^{-1}(\Omega)\right)$, then there exist a weak solution to problem (8).

Theorem 9. Under the assumptions of Theorem 8 with $d=2$, problem (8) has a unique weak solution.

Theorem 10 (periodic solutions). Under the assumptions of Theorem 8 there exist a pair of functions $(u, w)$ such that

$$
\begin{gathered}
u \in L^{\infty}(I ; H) \cap L^{4}\left(I ; V_{4}\right) \cap L^{4}(I ; V), \\
w \in L^{\infty}\left(I ; \mathbf{L}^{2}(\Omega)\right) \cap L^{2}\left(I ; \mathbf{H}_{0}^{1}(\Omega)\right), \\
\left(u^{\prime}(t), \varphi\right)+v a(u(t), \varphi)+v_{0}(\mathscr{K} u(t), \varphi)+\left(B_{u} u(t), \varphi\right) \\
=(\nabla \times w(t), \varphi)+(f(t), \varphi), \\
\left(w^{\prime}(t), \phi\right)+v_{1} a(w(t), \phi)+v_{1}(\nabla \cdot w(t), \nabla \cdot \phi) \\
+\left(B_{u} w(t), \phi\right)+\lambda_{1}(w(t), \phi) \\
=\lambda_{2}(\nabla \times u(t), \phi)+(g(t), \phi), \\
u(0)=u(T), \quad w(0)=w(T),
\end{gathered}
$$

$\forall \varphi \in V, \forall \phi \in \mathbf{H}_{0}^{1}(\Omega)$ in the sense of $D^{\prime}(0, T)$.

Theorem 11. Assuming that $d \leq 3, u_{0} \in V \cap V_{4}, w_{0} \in \mathbf{H}_{0}^{1}(\Omega)$, $f \in L^{4 / 3}\left(I ; V^{\prime}\right)$, and $g \in L^{2}\left(I ; \mathbf{H}^{-1}(\Omega)\right)$ there exist a unique weak solution to problem (8) such that

$$
\begin{gathered}
u^{\prime} \in L^{2}(I ; H), \\
u \in L^{\infty}\left(I ; V_{4}\right), \\
u \in L^{\infty}(I ; V), \\
w \in L^{\infty}\left(I ; \mathbf{H}_{0}^{1}(\Omega)\right), \\
w^{\prime} \in L^{2}\left(I ; \mathbf{L}^{2}(\Omega)\right) .
\end{gathered}
$$

\section{Proofs of the Results}

Proof of Theorem 8. We will show the existence of a weak solution to system (8) employing the Galerkin approximations. For that purpose we consider $\left(\varphi_{\nu}\right)_{v \in \mathbb{N}} \subset V$, a basis of eigenvectors of the Stokes operator and $\left(\phi_{\mu}\right)_{\mu \in \mathbb{N}} \subset \mathbf{H}_{0}^{1}(\Omega)$ a basis of eigenvectors of Lamé. We represent by $V_{m}=$ $\left[\varphi_{1}, \ldots, \varphi_{m}\right] \subset V$ the subspace generated by $\left\{\varphi_{1}, \ldots, \varphi_{m}\right\}$ and $W_{m}=\left[\phi_{1}, \ldots, \phi_{m}\right] \subset \mathbf{H}_{0}^{1}(\Omega)$ the subspace generated by $\left\{\phi_{1}, \ldots, \phi_{m}\right\}$. Let us also consider the pair $\left(u_{m}, w_{m}\right)$, such that

$$
\begin{aligned}
& u_{m}(x, t)=\sum_{r=1}^{m} g_{r m}(t) \varphi_{r}(x), \\
& w_{m}(x, t)=\sum_{r=1}^{m} h_{r m}(t) \phi_{r}(x)
\end{aligned}
$$

are the solution of the approximate problem

$$
\begin{gathered}
\left(u_{m}^{\prime}(t), \varphi_{r}\right)+v\left(A u_{m}(t), \varphi_{r}\right)+v_{0}\left(\mathscr{K} u_{m}(t), \varphi_{r}\right) \\
+\left\langle B_{u_{m}} u_{m}(t), \varphi_{r}\right\rangle=\left(\nabla \times w_{m}(t), \varphi_{r}\right)+\left(f(t), \varphi_{r}\right), \\
r=1, \ldots, m, \\
\left(w_{m}^{\prime}(t), \phi_{r}\right)-v_{1}\left(\nabla \cdot e(w), \phi_{r}\right)+\left\langle B_{u_{m}} w_{m}(t), \phi_{r}\right\rangle \\
+\lambda_{1}\left(w_{m}(t), \phi_{r}\right)=\lambda_{2}\left(\nabla \times u_{m}(t), \phi_{r}\right)+\left(g(t), \phi_{r}\right), \\
\quad r=1, \ldots, m, \\
u_{m}(0)=u_{0 m} \longrightarrow u_{0}, \quad \text { strongly in } H, \\
w_{m}(0)=w_{0 m} \longrightarrow w_{0}, \quad \text { strongly in } \mathbf{L}^{2}(\Omega) .
\end{gathered}
$$

The system of ordinary differential equations (37) has a local solution on an interval $\left[0, t_{m}\left[, 0<t_{m}<T\right.\right.$. The first estimate permits us to extend this solution to the whole interval $[0, T]$.

First Estimate. We sometimes omit the parameter $t$. Multiplying both sides of $(37)_{1}$ by $g_{r_{m}}$ and $(37)_{2}$ by $h_{r_{m}}$, next adding from $r=1$ to $r=m$, we obtain 


$$
\begin{aligned}
& \frac{1}{2} \frac{d}{d t}\left|u_{m}(t)\right|^{2}+v\left\|u_{m}(t)\right\|^{2} \\
& \quad+v_{0} \int_{\Omega} M\left(\left|e\left(u_{m}(t)\right)\right|^{2}\right)\left|e_{i j}\left(u_{m}(t)\right)\right|^{2} d x \\
& \leq\left|w_{m}(t)\right|\left\|u_{m}(t)\right\|+\|f(t)\|_{V^{\prime}}\left\|u_{m}(t)\right\|, \\
& \frac{1}{2} \frac{d}{d t}\left|w_{m}(t)\right|^{2}+v_{1}\left\|w_{m}(t)\right\|^{2} \\
& \quad+v_{1}\left|\nabla \cdot w_{m}(t)\right|^{2}+\lambda_{1}\left|w_{m}(t)\right|^{2} \\
& \leq \lambda_{2}\left\|u_{m}(t)\right\|\left|w_{m}(t)\right|+\|g(t)\|_{H^{-1}(\Omega)}\left\|w_{m}(t)\right\|,
\end{aligned}
$$

because $b(u, u, u)=b(u, w, w)=0$, for all $u \in V$, for all $w \in$ $\mathbf{H}_{0}^{1}(\Omega)$ (see Lions [3]), $\left|\nabla \times u_{m}\right|=\left|\nabla u_{m}\right|=\left\|u_{m}\right\|$ and $(\nabla \times$ $\left.w_{m}, u_{m}\right)=\left(w_{m}, \nabla \times u_{m}\right)$ (see Lukaszewicz [8]). Now using Young's inequality we obtain from (38) and (39), respectively:

$$
\begin{aligned}
\frac{1}{2} \frac{d}{d t}\left|u_{m}(t)\right|^{2}+v\left\|u_{m}(t)\right\|^{2} & \\
& +\frac{v_{0} c_{1} K}{2}\left\|u_{m}(t)\right\|_{V_{4}}^{4}+v_{2}\left\|u_{m}(t)\right\|^{4} \\
\leq & \frac{v}{4}\left\|u_{m}(t)\right\|^{2}+c_{\nu}\left|w_{m}(t)\right|^{2} \\
& +\frac{v_{2}}{2}\left\|u_{m}(t)\right\|^{4}+c_{v_{2}}\|f(t)\|_{V^{\prime}}^{4 / 3}, \\
\frac{1}{2} \frac{d}{d t} & \left|w_{m}(t)\right|^{2}+v_{1}\left\|w_{m}(t)\right\|^{2} \\
\leq & \frac{v}{4}\left\|u_{m}(t)\right\|^{2}+c_{\nu}\left|w_{m}(t)\right|^{2} \\
& +\frac{v_{1}}{2}\left\|w_{m}(t)\right\|^{2}+c_{v_{1}}\|g(t)\|_{H^{-1}(\Omega)}^{2} .
\end{aligned}
$$

From (27) (Korn's inequality) and (11) we can get

$$
\begin{aligned}
& \frac{v_{0}}{2} \int_{\Omega} M\left(\left|e\left(u_{m}\right)\right|^{2}\right)\left|e_{i j}\left(u_{m}\right)\right|^{2} d x \geq \frac{v_{0} c_{1}}{2}\left\|e\left(u_{m}\right)\right\|_{L^{4}(\Omega)}^{4} \\
& \quad \geq \frac{v_{0} c_{1} K}{2}\left\|u_{m}\right\|_{V_{4}}^{4}, \\
& \frac{v_{0}}{2} \int_{\Omega} M\left(\left|e\left(u_{m}\right)\right|^{2}\right)\left|e_{i j}\left(u_{m}\right)\right|^{2} d x \geq \frac{v_{0} c_{1}}{2}\left\|e\left(u_{m}\right)\right\|_{L^{4}(\Omega)}^{4} \\
& \quad \geq v_{2}\left\|u_{m}\right\|^{4} .
\end{aligned}
$$

Adding inequalities (40) and (41) and integrating from 0 to $t$, with $0 \leq t \leq T$, we conclude

$$
\begin{gathered}
\left(\left|u_{m}(t)\right|^{2}+\left|w_{m}(t)\right|^{2}\right)+v_{0} c_{1} K \int_{0}^{t}\left\|u_{m}(s)\right\|_{V_{4}}^{4} d s \\
\quad+v_{2} \int_{0}^{t}\left\|u_{m}(s)\right\|^{4} d s+v_{1} \int_{0}^{t}\left\|w_{m}(s)\right\|^{2} d s \\
\leq C+C \int_{0}^{t}\left(\left|u_{m}(s)\right|^{2}+\left|w_{m}(s)\right|^{2}\right) d s .
\end{gathered}
$$

By using Gronwall's inequality, we can write

$$
\left|u_{m}(t)\right|^{2}+\left|w_{m}(t)\right|^{2} \leq C .
$$

Therefore, it follows from (43) that

$$
\begin{gathered}
\left(u_{m}\right) \text { is bounded in } L^{\infty}(I ; H), \\
\left(u_{m}\right) \text { is bounded in } L^{4}\left(I ; V_{4}\right), \\
\left(u_{m}\right) \text { is bounded in } L^{4}(I ; V), \\
\left(w_{m}\right) \text { is bounded in } L^{\infty}\left(I ; \mathbf{L}^{2}(\Omega)\right), \\
\left(w_{m}\right) \text { is bounded in } L^{2}\left(I ; \mathbf{H}_{0}^{1}(\Omega)\right) .
\end{gathered}
$$

Second Estimate. We consider $P_{m}: V \rightarrow V_{m}$ as the orthogonal projections from $V$ to $V_{m}$ :

$$
P_{m} u=\sum_{j=1}^{m}\left(u, \varphi_{j}\right) \varphi_{j}, \quad \forall u(t) \in V .
$$

We also consider the adjoint operator to $P_{m}$ which is $P_{m}^{*}$ : $V^{\prime} \rightarrow V^{\prime}$. We note that $P_{m}^{*} u_{m}^{\prime}=u_{m}^{\prime}$. By the choice of the special basis $\left(\varphi_{v}\right)$, we obtain

$$
\left\|P_{m}\right\|_{\mathscr{L}(V, V)} \leq 1, \quad\left\|P_{m}^{*}\right\|_{\mathscr{L}\left(V^{\prime}, V^{\prime}\right)} \leq 1 .
$$

It follows from $(37)_{1},(21),(22)$, and (23) that

$$
u_{m}^{\prime}=-v P_{m}^{*} A u_{m}-v_{0} P_{m}^{*} \mathscr{K} u_{m}-P_{m}^{*} B u_{m}+P_{m}^{*} \nabla \times w_{m}+P_{m}^{*} f .
$$

We have $\left|\left\langle A u_{m}, v\right\rangle\right| \leq\left\|u_{m}\right\|\|v\|$, for all $u_{m}(t), v(t) \in V$. Therefore (47) implies

$$
\left(A u_{m}\right) \text { is bounded in } L^{4}\left(I ; V^{\prime}\right) \hookrightarrow L^{4 / 3}\left(I ; V^{\prime}\right) .
$$

Let $u_{m}(t), v(t) \in V$. From (20), Hölder's inequality, and (11) we take

$$
\begin{aligned}
\left|\left\langle\mathscr{K} u_{m}, v\right\rangle\right| & \leq\left|\left\langle M\left(\left|e\left(u_{m}\right)\right|^{2}\right) e\left(u_{m}\right), \nabla v\right\rangle\right| \leq c\left|e\left(u_{m}\right)\right|^{3}\|v\| \\
& \leq c\left|\nabla u_{m}\right|^{3}\|v\| \leq\left\|u_{m}\right\|^{3}\|v\| .
\end{aligned}
$$

Therefore, from (47), we obtain

$$
\left(\mathscr{K} u_{m}\right) \text { is bounded in } L^{4 / 3}\left(I ; V^{\prime}\right) .
$$

From $d \leq 3$ we derive $H_{0}^{1}(\Omega) \hookrightarrow L^{4}(\Omega)$. Using (17) and Hölder's inequality we conclude

$$
\left|\left\langle B_{u_{m}} u_{m}, v\right\rangle\right| \leq\left\|u_{m}\right\|_{L^{4}(\Omega)}\left\|u_{m}\right\|\|v\|_{L^{4}(\Omega)} \leq c\left\|u_{m}\right\|^{2}\|v\|
$$

for all $u_{m}(t), v(t) \in V$. Therefore, from (47)

$$
\left(B_{u_{m}} u_{m}\right) \text { is bounded in } L^{2}\left(I ; V^{\prime}\right) \hookrightarrow L^{4 / 3}\left(I ; V^{\prime}\right) .
$$


On the other hand, let $w_{m}(t) \in \mathbf{H}_{0}^{1}(\Omega)$, and $v(t) \in V$

$$
\left|\left\langle\nabla \times w_{m}, v\right\rangle\right|=\left|\left\langle w_{m}, \nabla \times v\right\rangle\right| \leq\left|w_{m}\right|\|v\| \leq c\left\|w_{m}\right\|\|v\|
$$

(see Lukaszewicz [8], pp. 116). It follows from (49) that

$$
\left(\nabla \times w_{m}\right) \text { is bounded in } L^{2}\left(I ; V^{\prime}\right) \hookrightarrow L^{4 / 3}\left(I ; V^{\prime}\right) \text {. }
$$

It follows from (53)-(59), (51), and hypothesis about $f$ that

$$
\left(u_{m}^{\prime}\right) \text { is bounded in } L^{4 / 3}\left(I ; V^{\prime}\right) \text {. }
$$

Analogously let $R_{m}: \mathbf{H}_{0}^{1}(\Omega) \rightarrow W_{m}$ be the orthogonal projections

$$
R_{m} w=\sum_{j=1}^{m}\left(w, \phi_{j}\right) \phi_{j}, \quad \forall w \in \mathbf{H}_{0}^{1}(\Omega)
$$

We also consider the adjoint operator to $R_{m}$, which is $R_{m}^{*}$ : $\mathbf{H}^{-1}(\Omega) \rightarrow \mathbf{H}^{-1}(\Omega)$. We have $R_{m}^{*} w_{m}^{\prime}=w_{m}^{\prime}$ and by the choice of the special basis $\left(\phi_{\nu}\right)$, we can get

$$
\left\|R_{m}\right\|_{\mathscr{L}\left(H_{0}^{1}(\Omega), H_{0}^{1}(\Omega)\right)} \leq 1, \quad\left\|R_{m}^{*}\right\|_{\mathscr{L}\left(H^{-1}(\Omega), H^{-1}(\Omega)\right)} \leq 1 .
$$

From $(37)_{2},(21),(22)$, and (23)

$$
\begin{aligned}
w_{m}^{\prime}= & -v_{1} R_{m}^{*} \nabla \cdot e\left(w_{m}\right)-R_{m}^{*} B w_{m}-\lambda_{1} R_{m}^{*} w_{m} \\
& +\lambda_{2} R_{m}^{*} \nabla \times u_{m}+R_{m}^{*} g .
\end{aligned}
$$

We note that $\left|\left\langle\nabla \cdot e\left(w_{m}\right), v\right\rangle\right|=\left|\left\langle\nabla w_{m}, \nabla v\right\rangle\right| \leq\left\|w_{m}\right\|\|v\|$, for all $w_{m}(t), v(t) \in \mathbf{H}_{0}^{1}(\Omega)$. Thus, (49) implies

$$
\left(\nabla \cdot e\left(w_{m}\right)\right) \text { is bounded in } L^{2}\left(I ; \mathbf{H}^{-1}(\Omega)\right) .
$$

Analogously and by using the embedding $H_{0}^{1}(\Omega) \hookrightarrow L^{2}(\Omega)$ we obtain

$$
\left(w_{m}\right) \text { is bounded in } L^{2}\left(I ; \mathbf{H}^{-1}(\Omega)\right) .
$$

On the other hand $\left|\left\langle\nabla \times u_{m}, v\right\rangle\right| \leq\left\|u_{m}\right\|\|v\|$, for all $u_{m}(t), v(t) \epsilon$ $\mathbf{H}_{0}^{1}(\Omega)$. Now, by using (47), we have

$$
\left(\nabla \times u_{m}\right) \text { is bounded in } L^{2}\left(I ; \mathbf{H}^{-1}(\Omega)\right) .
$$

Finally assuming that $d=2$, it follows from (18), (22), and Hölder's inequality that

$$
\begin{aligned}
\left|\left\langle B_{u_{m}} w_{m}, v\right\rangle\right| & =\left|b\left(u_{m}, w_{m}, v\right)\right|=\left|b\left(u_{m}, v, w_{m}\right)\right| \\
& \leq c\left\|u_{m}\right\|_{L^{4}(\Omega)}\|v\|\left\|w_{m}\right\|_{L^{4}(\Omega)}
\end{aligned}
$$

for all $u_{m}(t), w_{m}(t), v(t) \in \mathbf{H}_{0}^{1}(\Omega)$. Therefore, $\left\|B w_{m}\right\|_{H^{-1}(\Omega)}^{2} \leq$ $c\left|u_{m}\right|\left\|u_{m}\right\|\left|w_{m}\right|\left\|w_{m}\right\|$.

Because if $d=2$, then, $\|u\|_{L^{4}(\Omega)}^{2} \leq c|u|\|u\|$ (see Lions [3]). Now using Young's inequality we get

$$
\left\|B_{u_{m}} w_{m}\right\|_{H^{-1}(\Omega)}^{2} \leq c\left|u_{m}\right|^{2}\left\|u_{m}\right\|^{2}+c\left|w_{m}\right|^{2}\left\|w_{m}\right\|^{2} \text {. }
$$

Therefore, (47)-(49) and (45) permit us to obtain

$$
\left(B_{u_{m}} w_{m}\right) \text { is bounded in } L^{2}\left(I ; \mathbf{H}^{-1}(\Omega)\right), \quad d=2 .
$$

Analogously and assuming that $d=3$ we obtain from Lemma 7

$$
\left\|B_{u_{m}} w_{m}\right\|_{H^{-1}(\Omega)} \leq\left\|u_{m}\right\|_{L^{6}(\Omega)}\left|w_{m}\right|^{1 / 2}\left\|w_{m}\right\|^{1 / 2} .
$$

$H_{0}^{1}(\Omega) \hookrightarrow L^{6}(\Omega)$, because $d=3$. Thus,

$$
\begin{aligned}
\left\|B_{u_{m}} w_{m}\right\|_{H^{-1}(\Omega)}^{2} & \leq c\left\|u_{m}\right\|^{2}\left|w_{m}\right|\left\|w_{m}\right\| \\
& \leq c\left\|u_{m}\right\|^{4}+c\left|w_{m}\right|^{2}\left\|w_{m}\right\|^{2} .
\end{aligned}
$$

Therefore, (47)-(49) imply that

$$
\left(B_{u_{m}} w_{m}\right) \text { is bounded in } L^{2}\left(I ; \mathbf{H}^{-1}(\Omega)\right), \quad d=3 .
$$

It follows from (64)-(72) and hypothesis about $g$ that

$$
\left(w_{m}^{\prime}\right) \text { is bounded in } L^{2}\left(I ; \mathbf{H}^{-1}(\Omega)\right) \text {. }
$$

We note that (45)-(49), (60), (73), and the Aubin-Lions lemma imply that there exist subsequences of $\left(u_{m}\right)$ and $\left(w_{m}\right)$, still denoted by $\left(u_{m}\right)$ and $\left(w_{m}\right)$, such that

$$
\begin{gathered}
u_{m} \longrightarrow u \text { strongly in } L^{2}(I ; H), \quad \text { a.e. in } Q_{T}, \\
u_{m} \longrightarrow u \text { weak star in } L^{\infty}(I ; H), \\
u_{m} \longrightarrow u \text { weakly in } L^{4}(I ; V), \\
u_{m}^{\prime} \longrightarrow u^{\prime} \text { weakly in } L^{4 / 3}\left(I ; V^{\prime}\right),
\end{gathered}
$$

$w_{m} \longrightarrow w$ strongly in $L^{2}\left(I ; \mathbf{L}^{2}(\Omega)\right), \quad$ a.e. in $Q_{T}$,

$$
\begin{gathered}
w_{m} \longrightarrow w \text { weak star in } L^{\infty}\left(I ; \mathbf{L}^{2}(\Omega)\right), \\
w_{m} \longrightarrow w \text { weakly in } L^{2}\left(I ; \mathbf{H}_{0}^{1}(\Omega)\right), \\
w_{m}^{\prime} \longrightarrow w^{\prime} \text { weakly in } L^{2}\left(I ; H^{-1}(\Omega)\right), \\
\mathscr{K} u_{m} \longrightarrow \chi \text { weakly in } L^{4 / 3}\left(I ; V^{\prime}\right) .
\end{gathered}
$$

We note that (46) and (60) imply that $u \in C^{0}(I ; \mathbf{H})$. Similarly, (49) and (73) imply that $w \in C^{0}\left(I ; \mathbf{L}^{2}(\Omega)\right)$. Thus, it does make sense to consider $u(0)=u_{0}$ and $w(0)=w_{0}$.

In order to prove that

$$
\begin{gathered}
\int_{0}^{T} b\left(u_{m}, u_{m}, \varphi\right) \longrightarrow \int_{0}^{T} b(u, u, \varphi), \quad \forall \varphi \in \mathscr{D}(I ; \mathscr{V}), \\
\int_{0}^{T} b\left(u_{m}, w_{m}, \phi\right) \longrightarrow \int_{0}^{T} b(u, w, \phi), \quad \forall \phi \in \mathscr{D}(I ; \mathscr{D}(\Omega)),
\end{gathered}
$$

we use (74) and (78) (see [1], pp. 210). Now, we note that

$$
\int_{Q_{T}} e_{i j}\left(w_{m}\right) e_{i j}(\phi) d x d t \longrightarrow \int_{Q_{T}} e_{i j}(w) e_{i j}(\phi) d x d t,
$$


or equivalently

$$
\int_{0}^{T}\left\langle\nabla \cdot e\left(w_{m}\right), \phi\right\rangle d t \longrightarrow \int_{0}^{T}\langle\nabla \cdot e(w), \phi\rangle d t
$$

results from (80). The other terms of $(37)_{2}$ are obtained in the usual manner. In order to prove that

$$
\begin{aligned}
& \int_{Q_{T}} M\left(\left|e\left(u_{m}\right)\right|^{2}\right) e_{i j}\left(u_{m}\right) e_{i j}(\varphi) d x d t \\
& \longrightarrow \int_{Q_{T}} M\left(|e(u)|^{2}\right) e_{i j}(u) e_{i j}(\varphi) d x d t,
\end{aligned}
$$

we use the fact $\nabla u_{m} \rightarrow \nabla u$ a.e. in $Q_{T}$, (see [5] pp. 565-566). Therefore,

$$
\left|\nabla u_{m}\right|^{2} \longrightarrow|\nabla u|^{2} \quad \text { a.e. in } Q_{T}
$$

that is,

$$
\left|e\left(u_{m}\right)\right|^{2} \longrightarrow|e(u)|^{2} \quad \text { a.e. in } Q_{T} \text {. }
$$

Since $M \in C^{1}(0, \infty)$ we obtain from (88)

$$
M\left(\left|e\left(u_{m}\right)\right|^{2}\right) \longrightarrow M\left(|e(u)|^{2}\right) \quad \text { a.e. in } Q_{T} \text {. }
$$

Thus,

$$
M\left(\left|e\left(u_{m}\right)\right|^{2}\right) e_{i j}\left(u_{m}\right) e_{i j}(\varphi) \longrightarrow M\left(|e(u)|^{2}\right) e_{i j}(u) e_{i j}(\varphi)
$$

a.e. in $Q_{T}$ and for all $\varphi \in \mathscr{D}(I ; \mathscr{D}(\Omega))$. Using (46) and (11) we obtain

$$
\begin{aligned}
& \int_{Q_{T}} M\left(\left|e\left(u_{m}\right)\right|^{2}\right) e_{i j}\left(u_{m}\right) e_{i j}(\varphi) d x d t \\
& \leq C \int_{Q_{T}}\left|e\left(u_{m}\right)\right|^{3}\left|e_{i j}(\varphi)\right| d x d t \\
& \leq C \int_{Q_{T}}\left|e\left(u_{m}\right)\right|^{3} d x d t \leq C \int_{Q_{T}}\left|\nabla u_{m}\right|^{3} d x d t \leq C .
\end{aligned}
$$

It follows that

$$
M\left(\left|e\left(u_{m}\right)\right|^{2}\right) e_{i j}\left(u_{m}\right) e_{i j}(\varphi) \in L^{1}\left(Q_{T}\right) .
$$

Moreover, if $H \subset Q_{T}$ is a measurable set, we have from (11), (46), and Hölder's inequality that

$$
\begin{aligned}
& \int_{H} M\left(\left|e\left(u_{m}\right)\right|^{2}\right) e_{i j}\left(u_{m}\right) e_{i j}(\varphi) d x d t \\
& \quad \leq c \int_{H}\left|e\left(u_{m}\right)\right|^{3}|e(\varphi)| d x d t \\
& \quad \leq c\left(\int_{Q_{T}}\left|e\left(u_{m}\right)\right|^{4} d x d t\right)^{3 / 4}\left(\int_{H}|e(\varphi)|^{4} d x d t\right)^{1 / 4} \\
& \quad \leq c\left(\int_{Q_{T}}\left|\nabla u_{m}\right|^{4} d x d t\right)^{3 / 4}|H|^{1 / 4} \leq c|H|^{1 / 4} .
\end{aligned}
$$

Therefore,

$$
\sup _{m \in \mathbb{N}} \int_{H} M\left(\left|e\left(u_{m}\right)\right|^{2}\right) e_{i j}\left(u_{m}\right) e_{i j}(\varphi) d x d t \leq c|H|^{1 / 4}
$$

Assuming that $|H|$ is sufficiently small, we obtain

$$
\sup _{m \in \mathbb{N}} \int_{H} M\left(\left|e\left(u_{m}\right)\right|^{2}\right) e_{i j}\left(u_{m}\right) e_{i j}(\varphi) d x d t \leq \varepsilon,
$$

for all $\varepsilon \in \mathbb{R}$. Now using (90), (92), (95), and Vitali's lemma we can derive (86). Therefore, we can write $\chi=\mathscr{K} u$ in $L^{4 / 3}\left(I ; \mathbf{H}^{-1}(\Omega)\right)$. The convergences (74)-(82) and (85) and (86) allow us to pass the limit on system (37), with $\varphi_{r}$ and $q_{r}$ being fixed to obtain

$$
\begin{gathered}
u^{\prime}+v A u+v_{0} \mathscr{K} u+B_{u} u=\nabla \times w+f \quad \text { in } L^{4 / 3}\left(I ; V^{\prime}\right), \\
w^{\prime}+v_{1} \nabla \cdot e(w)+B_{u} w+\lambda_{1} w \\
=\lambda_{2} \nabla \times u+g \quad \text { in } L^{2}\left(I ; \mathbf{H}^{-1}(\Omega)\right) .
\end{gathered}
$$

This concludes the proof of Theorem 8 .

Proof of Theorem 9. Let $\left(u_{1}, w_{1}\right)$ and $\left(u_{2}, w_{2}\right)$ be weak solutions to Problem (8). Then,

$$
\begin{aligned}
& u_{1}, u_{2} \in L^{\infty}(I ; H) \cap L^{4}(I ; V) \cap L^{4}\left(I, V_{4}\right), \\
& w_{1}, w_{2} \in L^{\infty}\left(I ; \mathbf{L}^{2}(\Omega)\right) \cap L^{\infty}\left(I ; \mathbf{H}_{0}^{1}(\Omega)\right) .
\end{aligned}
$$

Consider $\tilde{u}=u_{1}-u_{2}$ and $\widetilde{w}=w_{1}-w_{2}$. Then, $(\tilde{u}, \widetilde{w})$ satisfies

$$
\begin{gathered}
\widetilde{u}^{\prime}+v A \widetilde{u}+v_{0}\left(\mathscr{K} u_{1}-\mathscr{K} u_{2}\right)+\left(B_{u_{1}} u_{1}-B_{u_{2}} u_{2}\right)=\nabla \times \widetilde{w} \\
\widetilde{w}^{\prime}+v_{1} A \widetilde{w}+v_{1} \nabla(\nabla \cdot \widetilde{w})+\left(B_{u_{1}} w_{1}-B_{u_{2}} w_{2}\right) \\
+\lambda_{1} \widetilde{w}=\lambda_{2} \nabla \times \widetilde{u} \\
\widetilde{u}(0)=\widetilde{w}(0)=0
\end{gathered}
$$

where the first equality has been considered in $L^{4 / 3}\left(I ; V^{\prime}\right)$ and the second in $L^{2}\left(\mathbf{H}^{-1}(\Omega)\right)$. We take the duality in $(98)_{1}$ and $(98)_{2}$ with $\widetilde{u}$ and $\widetilde{w}$, respectively, to obtain

$$
\begin{gathered}
\frac{1}{2} \frac{d}{d t}|\widetilde{u}|^{2}+v\|\widetilde{u}\|^{2}+v_{0}\left\langle\mathscr{K} u_{1}-\mathscr{K} u_{2}, \widetilde{u}\right\rangle \\
+\left\langle B_{u_{1}} u_{1}-B_{u_{2}} u_{2}, \widetilde{u}\right\rangle=\langle\nabla \times \widetilde{w}, \widetilde{u}\rangle, \\
\frac{1}{2} \frac{d}{d t}|\widetilde{w}|^{2}+v_{1}\|\widetilde{w}\|^{2}+v_{1}|\nabla \cdot \widetilde{w}|^{2} \\
+\left\langle B_{u_{1}} w_{1}-B_{u_{2}} w_{2}, \widetilde{w}\right\rangle+\lambda_{1}|\widetilde{w}|^{2}=\lambda_{2}\langle\nabla \times \widetilde{u}, \widetilde{w}\rangle, \\
\widetilde{u}(0)=\widetilde{w}(0)=0 .
\end{gathered}
$$


We note that

$$
\begin{aligned}
\left\langle B_{u_{1}} u_{1}(t)-B_{u_{2}} u_{2}(t), \widetilde{u}(t)\right\rangle & =b\left(\widetilde{u}(t), u_{1}(t), \widetilde{u}(t)\right), \\
\left\langle B_{u_{1}} w_{1}(t)-B_{u_{2}} w_{2}(t), \widetilde{w}(t)\right\rangle & =b\left(\widetilde{u}(t), w_{2}(t), \widetilde{w}(t)\right) .
\end{aligned}
$$

From the monotonicity of $\mathscr{K}$ we have $\left\langle\mathscr{K} u_{1}-\mathscr{K} u_{2}, \widetilde{u}\right\rangle \geq 0$. Thus,

$$
\begin{aligned}
& \frac{1}{2} \frac{d}{d t}|\widetilde{u}|^{2}+v\|\widetilde{u}\|^{2}+b\left(\widetilde{u}(t), u_{1}(t), \widetilde{u}(t)\right) \leq\langle\nabla \times \widetilde{w}, \widetilde{u}\rangle, \\
& \frac{1}{2} \frac{d}{d t}|\widetilde{w}|^{2}+v_{1}\|\widetilde{w}\|^{2}+\lambda_{1}|\widetilde{w}|^{2}+b\left(\widetilde{u}(t), w_{2}(t), \widetilde{w}(t)\right) \\
& \quad \leq \lambda_{2}\langle\nabla \times \widetilde{u}, \widetilde{w}\rangle .
\end{aligned}
$$

Adding the inequalities above, we derive

$$
\begin{aligned}
& \frac{1}{2} \frac{d}{d t}\left(|\widetilde{u}|^{2}+|\widetilde{w}|^{2}\right)+v\|\widetilde{u}\|^{2}+v_{1}\|\widetilde{w}\|^{2} \\
& \leq|\widetilde{w}|\|\widetilde{u}\|+\lambda_{2}\|\widetilde{u}\||\widetilde{w}| \\
& \quad+\left|b\left(\widetilde{u}, u_{1}, \widetilde{u}\right)\right|+\left|b\left(\widetilde{u}, w_{2}, \widetilde{w}\right)\right| .
\end{aligned}
$$

In other words,

$$
\begin{aligned}
\frac{1}{2} \frac{d}{d t} & \left(|\widetilde{u}|^{2}+|\widetilde{w}|^{2}\right)+v\|\widetilde{u}\|^{2}+v_{1}\|\widetilde{w}\|^{2} \\
\leq & \frac{v}{4}\|\widetilde{u}\|^{2}+\frac{v}{4}\|\widetilde{u}\|^{2}+c_{\nu}|\widetilde{w}|^{2} \\
& +\left|b\left(\widetilde{u}, u_{1}, \widetilde{u}\right)\right|+\left|b\left(\widetilde{u}, w_{2}, \widetilde{w}\right)\right| .
\end{aligned}
$$

Considering $d=2$, we get $H_{0}^{1}(\Omega) \hookrightarrow L^{4}(\Omega)$. Moreover (see Lions [3])

$$
|u|_{L^{4}(\Omega)} \leq c|u|^{1 / 2}\|u\|^{1 / 2} .
$$

Thus, using (17), Hölder's inequality, (104), and Young's inequality we take

$$
\begin{aligned}
\left|b\left(\widetilde{u}, u_{1}, \widetilde{u}\right)\right|+\left|b\left(\widetilde{u}, w_{2}, \widetilde{w}\right)\right| & \\
\leq & \|\widetilde{u}\|_{L^{4}(\Omega)}^{2}\left\|u_{1}\right\|+\|\widetilde{u}\|_{L^{4}(\Omega)}\left\|w_{2}\right\|\|\widetilde{w}\|_{L^{4}(\Omega)} \\
\leq & c\left\|u_{1}\right\||\widetilde{u}|\|\widetilde{u}\|+\left\|w_{2}\right\||\widetilde{u}|^{1 / 2}\|\widetilde{u}\|^{1 / 2}|\widetilde{w}|^{1 / 2}\|\widetilde{w}\|^{1 / 2} \\
\leq & \frac{v}{4}\|\widetilde{u}\|^{2}+c_{v}\left\|u_{1}\right\|^{2}|\widetilde{u}|^{2} \\
& +\sqrt{\frac{\nu}{2}}\|\widetilde{u}\| \sqrt{2 v_{1}}\|\widetilde{w}\|+c|\widetilde{u}||\widetilde{w}|\left\|w_{2}\right\|^{2} \\
\leq & \frac{v}{4}\|\widetilde{u}\|^{2}+c_{v}\left\|u_{1}\right\|^{2}|\widetilde{u}|^{2}+\frac{v}{4}\|\widetilde{u}\|^{2}+v_{1}\|\widetilde{w}\|^{2} \\
& +c\left\|w_{2}\right\|^{2}|\widetilde{u}|^{2}+c\left\|w_{2}\right\|^{2}|\widetilde{w}|^{2} .
\end{aligned}
$$

It follows from (103) that we can write

$$
\frac{1}{2} \frac{d}{d t}\left(|\widetilde{u}|^{2}+|\widetilde{w}|^{2}\right) \leq c\left(1+\left\|u_{1}\right\|^{2}+\left\|w_{2}\right\|^{2}\right)\left(|\widetilde{u}|^{2}+|\widetilde{w}|^{2}\right) .
$$

Integrating from 0 to $t$ we obtain

$$
\begin{aligned}
& |\widetilde{u}(t)|^{2}+|\widetilde{w}(t)|^{2} \\
& \quad \leq c \int_{0}^{t}\left(1+\left\|u_{1}(s)\right\|^{2}+\left\|w_{2}(s)\right\|^{2}\right)\left(|\widetilde{u}(s)|^{2}+|\widetilde{w}(s)|^{2}\right) d s .
\end{aligned}
$$

Applying Gronwall's inequality in (107), we deduce by using (47) and (49) that

$$
u_{1}(t)=u_{2}(t), \quad w_{1}(t)=w_{2}(t), \quad \forall t \in[0, T] .
$$

Theorem 9 has been proved.

Proof of Theorem 10. Under the assumptions and notations defined in the proof of the Theorem 8 we know that the system (37) has a solution whatever initial value $\left(u_{m}(0), w_{m}(0)\right) \in V_{m} \times W_{m}$. To proveTheorem 10, we first show that there exist an approximate solution for (37), such that

$$
\left(u_{m}(0), w_{m}(0)\right)=\left(u_{m}(T), w_{m}(T)\right)
$$

For this purpose, let us take $\varphi=u_{m}$ and $\phi=w_{m}$ in (37) to obtain

$$
\begin{aligned}
& \frac{1}{2} \frac{d}{d t}\left|u_{m}\right|^{2}+v\left\|u_{m}\right\|^{2}+v_{0} \int_{\Omega} M\left(\left|e\left(u_{m}\right)\right|^{2}\right)\left|e_{i j}\left(u_{m}\right)\right|^{2} d x \\
& \quad \leq\left|w_{m}\right|\left\|u_{m}\right\|+\|f\|_{V^{\prime}}\left\|u_{m}\right\| \\
& \frac{1}{2} \frac{d}{d t}\left|w_{m}\right|^{2}+v_{1} \int_{\Omega}\left|e_{i j}\left(w_{m}\right)\right|^{2} d x+\lambda_{1}\left|w_{m}\right|^{2} \\
& \quad \leq \lambda_{2}\left\|u_{m}\right\|\left|w_{m}\right|+|g|\left|w_{m}\right|,
\end{aligned}
$$

because $b(u, u, u)=0$ (see Lions [3]), $\left|\nabla \times u_{m}\right|=\left|\nabla u_{m}\right|=$ $\left\|u_{m}\right\|$, and $\left(\nabla \times w_{m}, u_{m}\right)=\left(w_{m}, \nabla \times u_{m}\right)$ (see Lukaszewicz [8]). Using (11) and (10) and (27) (Korn's inequality), we obtain from (110) and (111), respectively:

$$
\begin{gathered}
\frac{1}{2} \frac{d}{d t}\left|u_{m}\right|^{2}+v\left\|u_{m}\right\|^{2}+v_{0} M_{0}\left|e\left(u_{m}\right)\right|^{2} \\
\leq\left|w_{m}\right|\left\|u_{m}\right\|+\|f\|_{V^{\prime}}\left\|u_{m}\right\|, \\
\frac{1}{2} \frac{d}{d t}\left|w_{m}\right|^{2}+v_{1} K\left\|w_{m}\right\|^{2} \leq \lambda_{2}\left\|u_{m}\right\|\left|w_{m}\right|+|g|\left|w_{m}\right| .
\end{gathered}
$$

After usual computations, we can derive

$$
\begin{aligned}
& \frac{d}{d t}\left(\left|u_{m}\right|^{2}+\left|w_{m}\right|^{2}\right)+v\left\|u_{m}\right\|^{2}+v_{1} K\left\|w_{m}\right\|^{2} \\
& \leq C\left(\left|u_{m}\right|^{2}+\left|w_{m}\right|^{2}+\|f\|_{V^{\prime}}^{2}+|g|^{2}\right) .
\end{aligned}
$$

Considering the embeddings $V \hookrightarrow H$ and $\mathbf{H}_{0}^{1}(\Omega) \hookrightarrow \mathbf{L}^{2}(\Omega)$, there exists a constant $c_{2}$ such that

$$
\begin{gathered}
\frac{d}{d t}\left(\left|u_{m}\right|^{2}+\left|w_{m}\right|^{2}\right)+c_{2}\left|u_{m}\right|^{2}+c_{2}\left|w_{m}\right|^{2} \\
\quad \leq C\left(\left|u_{m}\right|^{2}+\left|w_{m}\right|^{2}+\|f\|_{V^{\prime}}^{2}+|g|^{2}\right) .
\end{gathered}
$$


Multiplying by $e^{c_{2} t}$ and integrating on $[0, t)$, we obtain

$$
\begin{gathered}
\left|u_{m}(t)\right|^{2}+\left|w_{m}(t)\right|^{2} \leq e^{-c_{2} t}\left(\left|u_{m}(0)\right|^{2}+\left|w_{m}(0)\right|^{2}\right) \\
+C+C \int_{0}^{t}\left(\left|u_{m}(s)\right|^{2}+\left|w_{m}(s)\right|^{2}\right) d s
\end{gathered}
$$

By Gronwall's inequality, we can write

$$
\left|u_{m}(t)\right|^{2}+\left|w_{m}(t)\right|^{2} \leq e^{-c_{2} t}\left(\left|u_{m}(0)\right|^{2}+\left|w_{m}(0)\right|^{2}\right)+C,
$$

for all $t \in[0, T]$. Let $\theta(t)=e^{-c_{2} t}$; we have $0<\theta(t)<1$. Thus,

$$
\left|u_{m}(T)\right|^{2}+\left|w_{m}(T)\right|^{2} \leq \theta\left(\left|u_{m}(0)\right|^{2}+\left|w_{m}(0)\right|^{2}\right)+C
$$

where $\theta=\theta(t)$ is a positive constant, such that $0<1-\theta<1$. Therefore, $C<C /(1-\theta)$. Taking $R>0$, such that $C /(1-$ $\theta)<R^{2}$, we obtain $C<(1-\theta) R^{2}$. Choosing the initial data $\left(u_{m}(0), w_{m}(0)\right) \in V_{m} \times W_{m}$, such that

$$
\left|u_{m}(0)\right|^{2}<\frac{R^{2}}{2}, \quad\left|w_{m}(0)\right|^{2}<\frac{R^{2}}{2} .
$$

We obtain from (118)

$$
\begin{gathered}
\left|u_{m}(T)\right|^{2}+\left|w_{m}(T)\right|^{2} \leq \theta\left(\left|u_{m}(0)\right|^{2}+\left|w_{m}(0)\right|^{2}\right) \\
+C<\theta R^{2}+(1-\theta) R^{2}=R^{2} .
\end{gathered}
$$

Therefore, $\left|u_{m}(0)\right|^{2}+\left|w_{m}(0)\right|^{2}<R^{2}$ implies that $\left|u_{m}(T)\right|^{2}+$ $\left|w_{m}(T)\right|^{2}<R^{2}$.

Now we define $\sigma: \mathscr{B}_{R}(0) \cap\left(V_{m} \times W_{m}\right) \rightarrow \mathscr{B}_{R}(0) \cap\left(V_{m} \times\right.$ $\left.W_{m}\right)$, such that

$$
\sigma\left(u_{m}(0), w_{m}(0)\right)=\left(u_{m}(T), w_{m}(T)\right),
$$

where $\mathscr{B}_{R}(0)=\left\{(u, w) \in H \times \mathbf{L}^{2}(\Omega) ;|u|^{2}+|w|^{2}<R\right\}$. We note that $\sigma$ is a continuous function because the solution of the (37) depends continuously of the initial data. We also note that (118) implies $\sigma\left(\mathscr{B}_{R}(0)\right) \subset \mathscr{B}_{R}(0)$. Therefore, it follows from Brower fixed-point theorem that $\sigma$ has a fixed point:

$$
\left(u_{0 m}, w_{0 m}\right) \in B_{R}(0) \subset V_{m} \times W_{m} .
$$

In other words, $\sigma\left(u_{0 m}, w_{0 m}\right)=\left(u_{0 m}, w_{0 m}\right)$. Taking the initial data $\left(u_{0 m}, w_{0 m}\right)$ in (37), that is, $\left(u_{m}(0), w_{m}(0)\right)=\left(u_{0 m}, w_{0 m}\right)$, we obtain

$$
\left(u_{m}(0), w_{m}(0)\right)=\left(u_{m}(T), w_{m}(T)\right) .
$$

Therefore, (37) has a periodic solution. Next, we obtain estimates to $(37)$ with the initial data $\left(u_{0 m}, w_{0 m}\right)$ as in the proof of Theorem 8 . We obtain

$$
\begin{gathered}
u_{m} \longrightarrow u \text { weakly in } L^{4}\left(I ; V_{4}\right), \\
u_{m}^{\prime} \longrightarrow u^{\prime} \text { weakly in } L^{4 / 3}\left(I ; V^{\prime}\right), \\
w_{m} \longrightarrow w \text { weakly in } L^{2}\left(I ; \mathbf{H}_{0}^{1}(\Omega)\right), \\
w_{m}^{\prime} \rightarrow w^{\prime} \text { weakly in } L^{2}\left(I ; \mathbf{H}^{-1}(\Omega)\right),
\end{gathered}
$$

where $u$ is the solution to problem (8) in the sense of Definition 3. The convergences (124) and (125) allow us to derive

$$
\int_{0}^{T} \frac{d}{d t}\left(u_{m}(s), v\right) \theta(s) d s \longrightarrow \int_{0}^{T} \frac{d}{d t}(u(s), v) \theta(s) d s,
$$

for all $v \in V$ and $\theta \in \mathscr{D}(0, T)$, with $\theta(T)=0$. In other words,

$$
\left(u_{m}(0), v\right) \longrightarrow(u(0), v), \quad \forall v \in V .
$$

The same argument with $\theta \in \mathscr{D}(0, T)$ and $\theta(0)=0$ allows us to derive

$$
\left(u_{m}(T), v\right) \longrightarrow(u(T), v), \quad \forall v \in V .
$$

It follows from (129) and (130) that $u(0)=u(T)$. Analogously, from (126) and (127), we obtain

$$
\begin{array}{ll}
\left(w_{m}(0), \bar{v}\right) \longrightarrow(w(0), \bar{v}), & \forall \bar{v} \in \mathbf{L}^{2}(\Omega), \\
\left(w_{m}(T), \bar{v}\right) \longrightarrow(w(T), \bar{v}), & \forall \bar{v} \in \mathbf{L}^{2}(\Omega) .
\end{array}
$$

Therefore, $w(0)=w(T)$. Theorem 10 has been proved.

Proof of Theorem 11. Let us consider $\tau: \mathbb{R}_{\text {sym }}^{d^{2}} \rightarrow \mathbb{R}_{\text {sym }}^{d^{2}}$ and the corresponding potential $\Phi: \mathbb{R}_{0}^{+} \rightarrow \mathbb{R}_{0}^{+}$such that

$$
\begin{gathered}
\tau(D)=M\left(|D|^{2}\right) D, \quad \forall D \in \mathbb{R}_{\text {sym }}^{d^{2}}, \\
\Phi\left(|D|^{2}\right)=\int_{0}^{|D|^{2}} M(s) d s, \quad \forall D \in \mathbb{R}_{\text {sym }}^{d^{2}}
\end{gathered}
$$

It is possible to verify (see Malek et al. [1]) that (132) satisfy the assumptions (28) of Lemma 5.

To obtain some estimate for $u_{m}^{\prime}$, we make $\varphi_{r}=u_{m}^{\prime}$ in $(37)_{1}$, to obtain applying Schwarz's inequality

$$
\begin{aligned}
\left|u_{m}^{\prime}\right|^{2} & +v \int_{\Omega} e_{i j}\left(u_{m}\right) e_{i j}\left(u_{m}^{\prime}\right) d x \\
& +v_{0} \int_{\Omega} \tau_{i j}\left(e\left(u_{m}\right)\right) e_{i j}\left(u_{m}^{\prime}\right) d x \\
\leq & \int_{\Omega}\left|u_{m_{i}}\right|\left|\frac{\partial u_{m_{j}}}{\partial x_{i}}\right|\left|u_{m_{j}}^{\prime}\right| d x \\
& +\left|u_{m}^{\prime}\right|\left|\nabla \times w_{m}\right|+|f|\left|u_{m}^{\prime}\right| .
\end{aligned}
$$

Remark 12. We note that (see [4])

$$
\int_{\Omega} \tau_{i j}(e(v)) e_{i j}\left(v^{\prime}\right) d x=\frac{d}{d t} \int_{\Omega} \Phi\left(|e(v)|^{2}\right) d x .
$$

Applying Young's inequality, (49) and (134), we obtain from (141) that

$$
\begin{aligned}
& \frac{1}{2}\left|u_{m}^{\prime}\right|^{2}+\frac{v}{2} \frac{d}{d t}\left|e\left(u_{m}\right)\right|^{2}+v_{0} \frac{d}{d t} \int_{\Omega} \Phi\left(\left|e\left(u_{m}\right)\right|^{2}\right) d x \\
& \quad \leq c+c \int_{\Omega}\left|u_{m}\right|^{2}\left|\nabla u_{m}\right|^{2} d x .
\end{aligned}
$$


We observe that (46) implies $u_{m}(t) \in V_{4} \hookrightarrow \mathbf{L}^{\infty}(\Omega)$ because $d \leq 3$. On the other hand, (47) implies $\nabla u_{m}(t) \in \mathbf{L}^{2}(\Omega)$. Thus, applying the Hölder's inequality in (135), integrating on $(0, t)$, with $t \leq T$, and after applying Korn's inequality, we obtain

$$
\begin{aligned}
& \int_{0}^{t}\left|u_{m}^{\prime}(s)\right|^{2} d s+v_{1} K\left\|u_{m}\right\|^{2}+2 v_{0} \int_{\Omega} \Phi\left(\left|e\left(u_{m}\right)\right|^{2}\right) d x \\
& \leq c+2 v_{0} \int_{\Omega} \Phi\left(\left|e\left(u_{m}(0)\right)\right|^{2}\right) d x+v_{1} K\left\|u_{m}(0)\right\|^{2} \\
& \quad+c \int_{0}^{t}\left|u_{m}(s)\right|_{L^{\infty}(\Omega)}^{2}\left\|u_{m}(s)\right\|^{2} d s .
\end{aligned}
$$

Observing that (46) implies $\left\|u_{m}(t)\right\|_{L^{\infty}(\Omega)}^{2} \in L^{2}(0, T)$ and also $\left\|u_{m}(t)\right\|^{2} \in L^{2}(0, T)$, because of $(47)$, so we can obtain by using Hölder's inequality in (136)

$$
\begin{aligned}
\int_{0}^{t}\left|u_{m}^{\prime}(s)\right|^{2} d s & +v_{1} K\left\|u_{m}\right\|^{2} \\
& +2 v_{0} \int_{\Omega} \Phi\left(\left|e\left(u_{m}\right)\right|^{2}\right) d x \leq c
\end{aligned}
$$

because (29) and $u_{0} \in V \cap V_{4}$. Now we note that (29) and Korn's inequality imply

$$
\int_{\Omega} \Phi\left(\left|e\left(u_{m}\right)\right|^{2}\right) d x \geq C_{3}\left\|e\left(u_{m}\right)\right\|_{L^{4}(\Omega)}^{4} \geq C_{3} K\left\|u_{m}\right\|_{V_{4}}^{4} .
$$

It follows from (137) that

$$
\int_{0}^{t}\left|u_{m}^{\prime}(s)\right|^{2} d s+v_{1} K\left\|u_{m}\right\|^{2}+C_{3} K\left\|u_{m}\right\|_{V_{4}}^{4} \leq c .
$$

Inequality (139) permits us to obtain the following estimates:

$$
\begin{aligned}
& \left(u_{m}^{\prime}\right) \text { is bounded in } L^{2}(I ; H), \\
& \left(u_{m}\right) \text { is bounded in } L^{\infty}(I ; V), \\
& \left(u_{m}\right) \text { is bounded in } L^{\infty}\left(I ; V_{4}\right) .
\end{aligned}
$$

Now we will obtain some estimate for $w_{m}^{\prime}$. For that purpose, we make $\phi_{r}=w_{m}^{\prime}$ in $(37)_{2}$ to obtain

$$
\begin{aligned}
& \left|w_{m}^{\prime}\right|^{2}+v_{1} \int_{\Omega} e_{i j}\left(w_{m}\right) e_{i j}\left(w_{m}^{\prime}\right) d x+\frac{\lambda_{1}}{2} \frac{d}{d t}\left|w_{m}\right|^{2} \\
& \quad \leq \int_{\Omega}\left|u_{m_{i}}\right|\left|\frac{\partial w_{m_{j}}}{\partial x_{i}}\right|\left|w_{m_{j}}^{\prime}\right| d x+\lambda_{2}\left\|u_{m}\right\|\left|w_{m}^{\prime}\right|+|g|\left|w_{m}^{\prime}\right| .
\end{aligned}
$$

Applying Young's inequality and using (47) we derive

$$
\begin{gathered}
\frac{1}{2}\left|w_{m}^{\prime}\right|^{2}+\frac{v_{1}}{2} \frac{d}{d t}\left|e\left(w_{m}\right)\right|^{2}+\frac{\lambda_{1}}{2} \frac{d}{d t}\left|w_{m}\right|^{2} \\
\leq c+c \int_{\Omega}\left|u_{m}\right|^{2}\left|\nabla w_{m}\right|^{2} d x .
\end{gathered}
$$

We observe that (46) implies $u_{m}(t) \in V_{4} \hookrightarrow \mathbf{L}^{\infty}(\Omega)$ because $d \leq 3$. On the other hand, (49) implies $\nabla w_{m}(t) \in \mathbf{L}^{2}(\Omega)$. Thus, applying Hölder's inequality in (142), integrating on $(0, t)$, with $t \leq T$, and after applying Korn's inequality, we obtain

$$
\begin{aligned}
& \int_{0}^{t}\left|w_{m}^{\prime}(s)\right|^{2} d s+v_{1} K\left\|w_{m}\right\|^{2} \\
& \quad \leq c+c \int_{0}^{t}\left|u_{m}(s)\right|_{L^{\infty}(\Omega)}^{2}\left\|w_{m}(s)\right\|^{2} d s
\end{aligned}
$$

because $w_{0} \in \mathbf{H}_{0}^{1}(\Omega)$. Furthermore (46) implies $\left\|u_{m}(t)\right\|_{L^{\infty}(\Omega)}^{2}$ $\in L^{2}(0, T)$ and $(49)$ implies $\left\|u_{m}(t)\right\| \in L^{1}(0, T)$. Thus, by using Gronwall's inequality in (143), we conclude

$$
\begin{aligned}
& \left(w_{m}^{\prime}\right) \text { is bounded in } L^{2}\left(I ; \mathbf{L}^{2}(\Omega)\right), \\
& \left(w_{m}\right) \text { is bounded in } L^{\infty}\left(I ; \mathbf{H}_{0}^{1}(\Omega)\right) .
\end{aligned}
$$

In order to prove uniqueness of solution to the case $d=3$ we observe that by assuming $d=3$ we have $H_{0}^{1} \hookrightarrow L^{6}(\Omega)$. From Lemma 7 with $s=4, r=6$ and Young's inequality we derive

$$
\begin{aligned}
& \left|b\left(\tilde{u}, u_{1}, \tilde{u}\right)\right|+\left|b\left(\widetilde{u}, w_{2}, \widetilde{w}\right)\right| \\
& \leq c\|\tilde{u}\|_{L^{6}(\Omega)}\left\|u_{1}\right\||\tilde{u}|^{1 / 2}\|\tilde{u}\|^{1 / 2} \\
& +c\|\widetilde{u}\|_{L^{6}(\Omega)}\left\|w_{2}\right\||\widetilde{w}|^{1 / 2}\|\widetilde{w}\|^{1 / 2} \\
& \leq c\left\|u_{1}\right\||\widetilde{u}|^{1 / 2}\|\widetilde{u}\|^{3 / 2} \\
& +c\left\|w_{2}\right\||\widetilde{w}|^{1 / 2}\|\widetilde{u}\|\|\widetilde{w}\|^{1 / 2} \\
& \leq \frac{v}{4}\|\tilde{u}\|^{2}+c_{\nu}\left\|u_{1}\right\|^{4}|\tilde{u}|^{2} \\
& +\frac{\nu}{4}\|\tilde{u}\|^{2}+c_{\nu}\left\|w_{2}\right\|^{2}|\widetilde{w}|\|\widetilde{w}\| \\
& \leq \frac{\nu}{4}\|\tilde{u}\|^{2}+c_{\nu}\left\|u_{1}\right\|^{4}|\widetilde{u}|^{2} \\
& +\frac{\nu}{4}\|\tilde{u}\|^{2}+v_{1}\|\widetilde{w}\|^{2}+c_{v_{1}}\left\|w_{2}\right\|^{4}|\widetilde{w}|^{2} .
\end{aligned}
$$

It follows from (103) that

$$
\frac{1}{2} \frac{d}{d t}\left(|\widetilde{u}|^{2}+|\widetilde{w}|^{2}\right) \leq c\left(1+\left\|u_{1}\right\|^{4}+\left\|w_{2}\right\|^{4}\right)\left(|\widetilde{u}|^{2}+|\widetilde{w}|^{2}\right) .
$$

Integrating from 0 to $t$ we obtain

$$
\begin{aligned}
& |\widetilde{u}(t)|^{2}+|\widetilde{w}(t)|^{2} \\
& \quad \leq c \int_{0}^{t}\left(1+\left\|u_{1}(s)\right\|^{4}+\left\|w_{2}(s)\right\|^{4}\right)\left(|\widetilde{u}(s)|^{2}+|\widetilde{w}(s)|^{2}\right) d s .
\end{aligned}
$$

Applying Gronwall's inequality in (148), we deduce by using (47) and (145) that

$$
u_{1}(t)=u_{2}(t), \quad w_{1}(t)=w_{2}(t), \quad \forall t \in[0, T] .
$$




\section{Conflict of Interests}

The authors declare that there is no conflict of interests regarding the publication of this paper.

\section{References}

[1] J. Malek, J. Necas, M. Rokyta, and M. RuzicKa, Weak and Measure-Valued Solutions to Evolutionary PDEs, Chapman \& Hall, New York, NY, USA, 1st edition, 1996.

[2] J. Malek, K. R. Rajagopal, and M. Ruzicka, "Existence and regularity of solution and stability of the rest state for fluids with shear dependent viscosity," Mathematical Models and Methods in Applied Sciences, vol. 5, no. 6, pp. 789-812, 1995.

[3] J. L. Lions, Quelques Methodes de Res olution Des Problemes Aux Limtes Non Lin eaires, Dunod, Paris, France, 1969.

[4] J. Malek, J. Necas, and M. Ruzicka, "On weak solutions to a class of non-Newtonian incompressible uids in bounded threedimensional domains: the case $p \geq 2$," Advances in Differential Equations, vol. 6, no. 3, pp. 257-302, 2001.

[5] J. Frehse and J. Málek, "Problems due to the no-slip boundary in incompressible fluid dynamics," in Geometric Analysis and Nonlinear Partial Differential Equations, pp. 559-571, Springer, Berlin, Germany, 2003.

[6] A. C. Eringen, “Theory of micropolar fluids," Journal of Mathematical Fluid Mechanics, vol. 16, pp. 1-18, 1966.

[7] C. Calmelet-Eluhu and D. R. Majumdar, "Flow of a micropolar fluid through a circular cylinder subject to longitudinal and torsional oscillations," Mathematical and Computer Modelling, vol. 27, no. 8, pp. 69-78, 1998.

[8] G. Lukaszewicz, Micropolar Fluids, Theory and Applications, Modeling and Simulations in Science, Engineering and Technology, Birkhauser, Boston, Mass, USA, 1999.

[9] H. W. Alt, Lineare Funktionalanalysis, Springer, Berlin, Germany, 2nd edition, 1992 (German). 


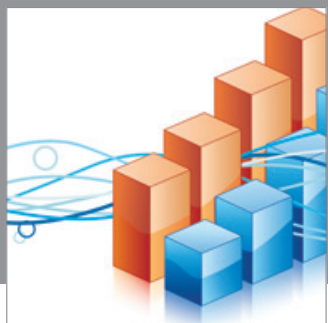

Advances in

Operations Research

mansans

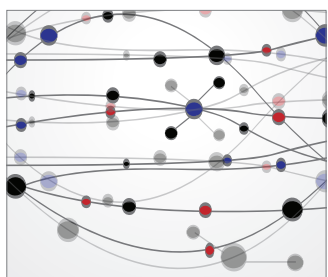

The Scientific World Journal
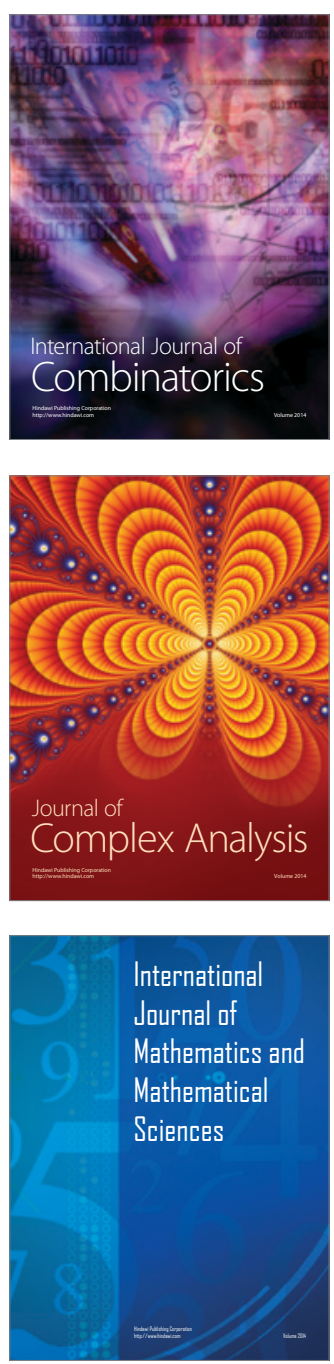
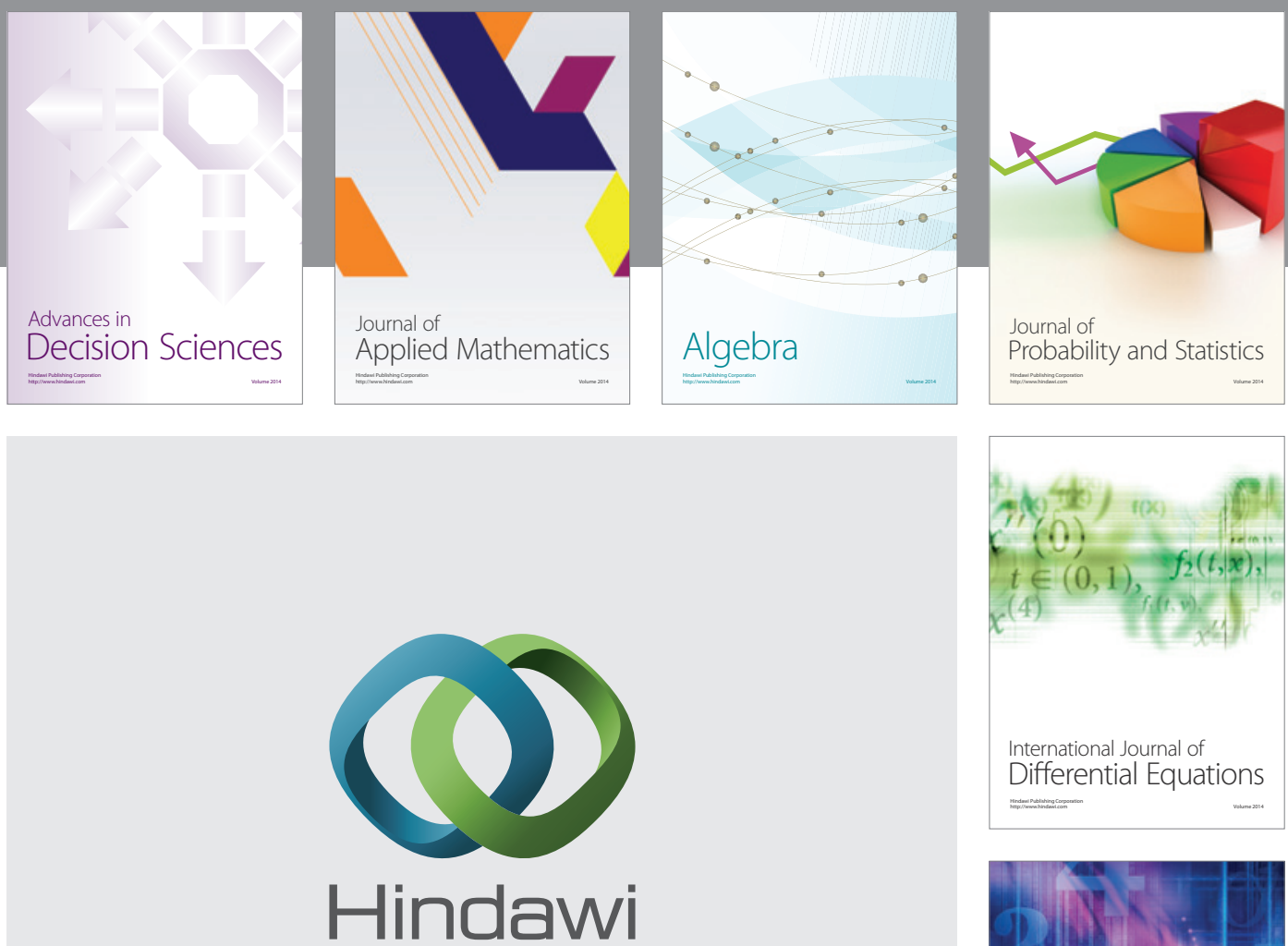

Submit your manuscripts at http://www.hindawi.com
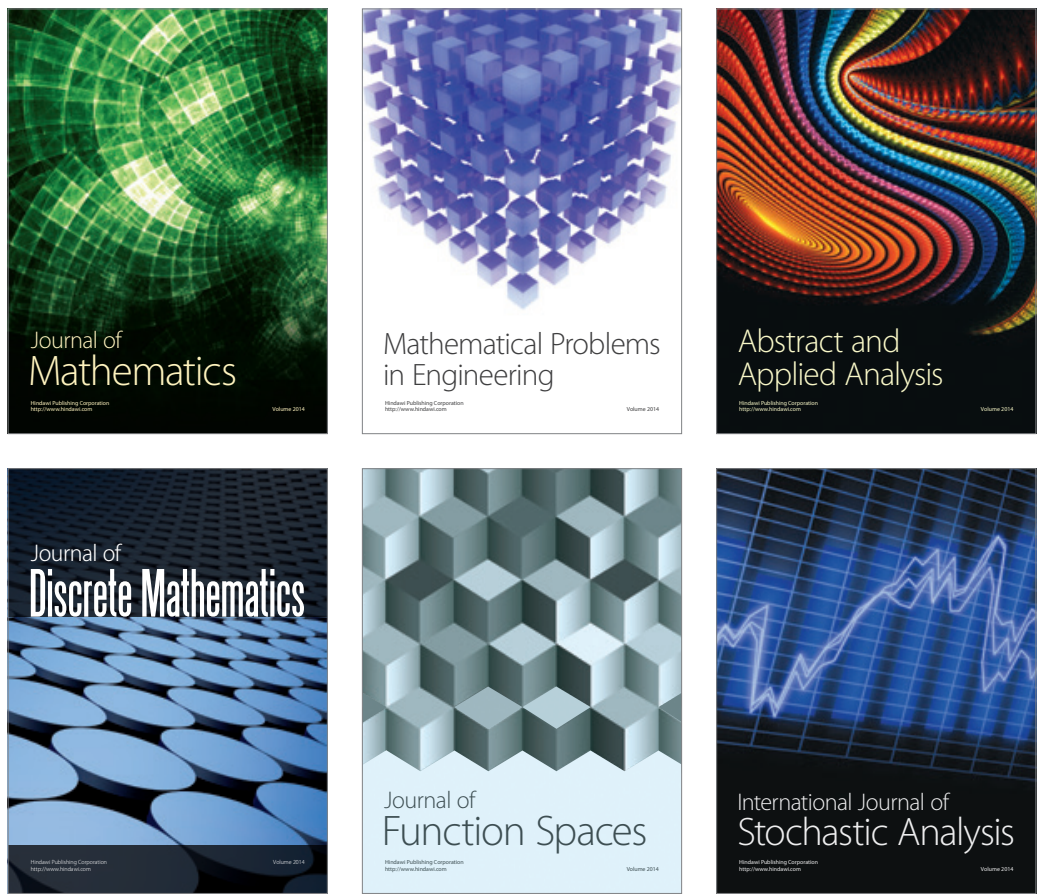

Journal of

Function Spaces

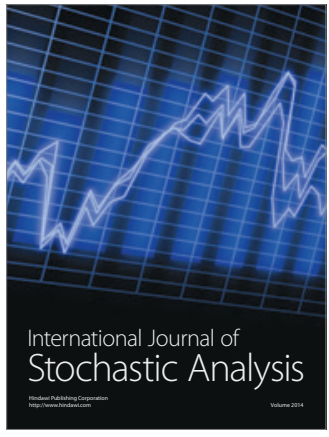

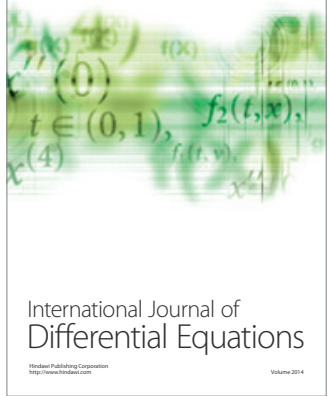
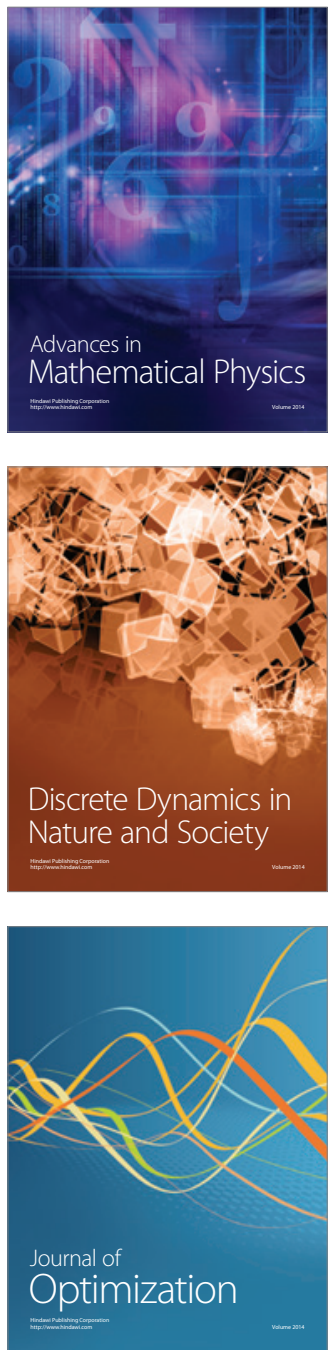